PROCEEDINGS OF THE

AMERICAN MATHEMATICAL SOCIETY

Volume 135, Number 7, July 2007, Pages 2065-2072

S 0002-9939(07)08691-1

Article electronically published on February 2, 2007

\title{
A NEW PROOF AND GENERALIZATIONS OF GEARHART'S THEOREM
}

\author{
VU QUOC PHONG \\ (Communicated by Carmen C. Chicone)
}

\begin{abstract}
Let $H$ be a Hilbert space, let $A P(\mathbf{R}, H)$ be the space of almost periodic functions from $\mathbf{R}$ to $H$, and let $A$ be a closed densely defined linear operator on $H$. For a closed subset $\Lambda \subset \mathbf{R}$, let $M(\Lambda)$ be the subspace of $A P(\mathbf{R}, H)$ consisting of functions with spectrum contained in $\Lambda$. We prove that the following properties are equivalent: (i) for every function $f \in M(\Lambda)$ there exists a unique mild solution $u \in M(\Lambda)$ of equation $u^{\prime}(t)=A u(t)+f(t)$; (ii) $i \Lambda \subset \rho(A)$ and $\sup _{\lambda \in \Lambda}\left\|(i \lambda-A)^{-1}\right\|<\infty$. The case $\Lambda=\{2 \pi k: k=$ $0, \pm 1, \pm 2, \ldots\}$ yields a new proof of the well-known Gearhart's spectral mapping theorem.
\end{abstract}

1.

Let $H$ be a Hilbert space and let $T(t), t \geq 0$, be a strongly continuous semigroup $\left(C_{0}\right.$-semigroup) of bounded linear operators on $H$, with the generator $A$. The following is the well-known Gearhart's spectral mapping theorem. It was proved by Gearhart [2] for contraction semigroups and later independently by Herbst [3], Howland [4] and Prüss [7] for $C_{0}$-semigroups (see also [6], p. 95).

Theorem 1. The following are equivalent:

(i) $1 \in \rho(T(1))$;

(ii) $2 \pi k i \in \rho(A)$ for every $k \in \mathbf{Z}$ and $\sup _{k \in \mathbf{Z}}\left\|(2 \pi k i-A)^{-1}\right\|<\infty$;

(iii) for every 1-periodic continuous function $f: \mathbf{R} \rightarrow H$, there exists a unique 1-periodic mild solution of the equation

$$
u^{\prime}(t)=A u(t)+f(t) .
$$

In this note, we prove the following generalization of this theorem. Note that the notion of almost periodic functions used in Theorem 2 is in the sense of Hilbert space (see Section 2 for a precise definition).

Theorem 2. Let $A$ be a closed densely defined linear operator on a Hilbert space $H$ and let $\Lambda$ be a closed subset of $\mathbf{R}$. Then the following are equivalent:

(i) For every almost periodic function $f: \mathbf{R} \rightarrow H$ such that $\sigma(f) \subset \Lambda$, there exists a unique almost periodic mild solution $u$ of (1) such that $\sigma(u) \subset \Lambda$;

Received by the editors December 29, 2005 and, in revised form, March 2, 2006.

2000 Mathematics Subject Classification. Primary 47D06, 35B40.

Key words and phrases. $C_{0}$-semigroup, almost periodic, admissible subspace, spectral mapping theorem.

(C)2007 American Mathematical Society Reverts to public domain 28 years from publication 
(ii) $i \Lambda \subset \rho(A)$ and

$$
\sup _{\lambda \in \Lambda}\left\|(i \lambda-A)^{-1}\right\|<\infty .
$$

Since $f$ is 1 -periodic if and only if $\sigma(f) \subset\{2 \pi k: k=0, \pm 1, \pm 2, \ldots\}$, the equivalence of (ii) and (iii) in Theorem 1 (which is the main part of the theorem) is a particular case of Theorem 2 .

Note that we do not assume that $A$ is a generator of a $C_{0}$-semigroup.

2.

Let $H$ be a Hilbert space with the inner product denoted by $(x, y)_{H}, x, y \in H$. Let $A P_{b}(\mathbf{R}, H)$ be the space of Bohr's almost periodic functions defined on $\mathbf{R}$ with values in $H$. In $A P_{b}(\mathbf{R}, H)$ the following limit (mean) exists:

$$
\langle f, g\rangle=M\{f, g\}:=\lim _{T \rightarrow \infty} \frac{1}{2 T} \int_{-T}^{T}(f(t), g(t))_{H} d t
$$

and defines an inner product. Thus, $A P_{b}(\mathbf{R}, H)$ is a pre-Hilbert space and its completion, denoted by $A P(\mathbf{R}, H)$, is a Hilbert space. Below we will denote the inner product and norm in $A P(\mathbf{R}, H)$ by $\langle,\rangle_{A P}$ and \|\|$_{A P}$, respectively.

In $A P(\mathbf{R}, H)$, the family of functions $e_{\lambda, x}(t)=e^{i \lambda t} x, \lambda \in \mathbf{R}$ and $x \in H$, form a complete system (which are orthogonal for different $\lambda$ 's). If $x_{\alpha}$ form an orthonormal basis in $H$, then $e_{\lambda, x_{\alpha}}(t)=e^{i \lambda t} x_{\alpha}$ form an orthonormal basis in $A P(\mathbf{R}, H)$.

For each $f \in A P(\mathbf{R}, H)$, the Fourier-Bohr transform is defined by

$$
a(\lambda, f)=\lim _{T \rightarrow \infty} \frac{1}{2 T} \int_{-T}^{T} f(t) e^{-i \lambda t} d t .
$$

The set $\sigma(f):=\{\lambda \in \mathbf{R}: a(\lambda, f) \neq 0\}$ is called the Bohr spectrum of $f$. It is well known that $\sigma(f)$ is (at most) countable. The Fourier-Bohr series of $f$ is

$$
\sum_{\lambda \in \sigma(f)} a(\lambda, f) e^{i \lambda t}
$$

and it converges to $f$ (in the topology of $A P(\mathbf{R}, H)$ ). Moreover, the following Parseval's equality holds:

$$
\|f\|_{A P}^{2}=\sum_{\lambda \in \sigma(f)}\|a(\lambda, f)\|_{H}^{2}, f \in A P(\mathbf{R}, H) .
$$

In the sequel, if a function $f$ in $A P(\mathbf{R}, H)$ has a Fourier-Bohr series

$$
\sum_{\lambda \in \sigma(f)} a(\lambda, f) e^{i \lambda t}
$$

then we will write

$$
f \sim \sum_{\lambda \in \sigma(f)} a(\lambda, f) e^{i \lambda t} .
$$

We will also frequently use the following equality, which is valid for every $u, v \in$ $A P(\mathbf{R}, H)$ :

$$
\langle u, v\rangle_{A P}=\sum_{\lambda}(a(\lambda, u), a(\lambda, v))_{H}
$$

(the sum is over a countable set of exponents $\lambda$ ). In particular,

$$
\left\langle u, e^{i \lambda t} x\right\rangle_{A P}=(a(\lambda, u), x)_{H} \text {, for all } u \in A P(\mathbf{R}, H), x \in H \text {. }
$$


Note that there is a family of orthogonal projections $P_{\lambda}, \lambda \in \mathbf{R}$, on $A P(\mathbf{R}, H)$ defined by $P(\lambda) f=e^{i \lambda t} a(\lambda, f)$, which satisfies $P_{\lambda} P_{\mu}=0$ if $\lambda \neq \mu$. It is clear that $H_{\lambda}:=P_{\lambda} H=\left\{e^{i \lambda t} x: x \in H\right\}$. The family $H_{\lambda}, \lambda \in \mathbf{R}$, is pairwise orthogonal and complete in $A P(\mathbf{R}, H)$. For these and other facts about almost periodic functions, we refer the reader to [5].

Consider the translation group $S(t),-\infty<t<\infty$, on $A P(\mathbf{R}, H)$. The operators $S(t)$ are first defined for functions $f$ in $A P_{b}(\mathbf{R}, H)$ by $(S(t) f)(\cdot)=f(\cdot+t)$, and extended to $A P(\mathbf{R}, H)$ by continuity. It is clear that $S(t)$ is a strongly continuous group of unitary operators. Let $\mathcal{D}$ be the generator of $S(t)$. Then $\mathcal{D}$ is a skew self-adjoint operator on $A P(\mathbf{R}, H)$, i.e. $\mathcal{D}^{*}=i \mathcal{D}$, and is the closure of the operator of differentiation, with the natural domain.

Let $A$ be a closed, densely defined linear operator on a Hilbert space $H$. The operator $A$ generates an operator $\mathcal{A}$ on $A P(\mathbf{R}, H)$ in a natural manner. Namely, we define $\mathcal{A}$ on $A P(\mathbf{R}, H)$ by

$$
\begin{gathered}
D(\mathcal{A}):=\{f \in A P(\mathbf{R}, H): a(\lambda, f) \in D(A) \text { for all } \lambda \in \sigma(f) \\
\text { and } \left.\sum_{\lambda \in \sigma(f)}\|A a(\lambda, f)\|_{H}^{2}<\infty\right\}
\end{gathered}
$$

and

$$
(\mathcal{A} f) \sim \sum_{\lambda \in \sigma(f)} A a(\lambda, f) e^{i \lambda t}, \text { for } f \in D(\mathcal{A}) .
$$

Lemma 3. $\mathcal{A}$ is a densely defined closed operator and $\sigma(\mathcal{A})=\sigma(A)$.

Proof. It is clear that $D(\mathcal{A})$ contains linear combinations of functions of the form $e^{i \lambda t} x$, with $\lambda \in \mathbf{R}$ and $x \in D(A)$. From this it is easily seen that $D(\mathcal{A})$ is dense in $A P(\mathbf{R}, H)$. This implies that $\mathcal{A}^{*}$ is well defined (and densely defined closed). Moreover, for every $f \in D(\mathcal{A})$ with the Fourier-Bohr series $\sum_{\lambda \in \sigma(f)} a(\lambda, f) e^{i \lambda t}$, and for every $x \in D\left(A^{*}\right)$, we have

$$
\left\langle\mathcal{A} f, e^{i \xi t} x\right\rangle=(A a(\xi, f), x)_{H}=\left(a(\xi, f), A^{*} x\right)_{H}=\left\langle f, e^{i \xi t} A^{*} x\right\rangle_{A P},
$$

which implies that $e^{i \xi t} x \in D\left(\mathcal{A}^{*}\right)$ and $\mathcal{A}^{*}\left(e^{i \xi t} x\right)=e^{i \xi t} A^{*} x$.

Now assume that $f_{n} \in D(\mathcal{A}), f_{n} \rightarrow f, \mathcal{A} f_{n} \rightarrow g$. We must show that $f \in D(\mathcal{A})$ and $\mathcal{A} f=g$. Let

$$
f \sim \sum a(\lambda, f) e^{i \lambda t}, \quad f_{n} \sim \sum a\left(\lambda, f_{n}\right) e^{i \lambda t}, \quad g \sim \sum a(\lambda, g) e^{i \lambda t} .
$$

Since $f_{n} \in D(\mathcal{A})$ and $A$ is closed, we have $a\left(\lambda, f_{n}\right) \in D(A)$ and $A a\left(\lambda, f_{n}\right) \rightarrow a(\lambda, g)$. Moreover, for every $h \in D\left(\mathcal{A}^{*}\right)$ we have

$$
\left\langle f, \mathcal{A}^{*} h\right\rangle_{A P}=\lim _{n \rightarrow \infty}\left\langle f_{n}, \mathcal{A}^{*} h\right\rangle_{A P}=\lim _{n \rightarrow \infty}\left\langle\mathcal{A} f_{n}, h\right\rangle_{A P}=\langle g, h\rangle_{A P}
$$

In particular, for every $x \in D\left(A^{*}\right)$, we have $h(t)=e^{i \lambda t} x \in D\left(\mathcal{A}^{*}\right), \mathcal{A}^{*} h=e^{i \lambda t} A^{*} x$ and

$$
\left\langle f, \mathcal{A}^{*} h\right\rangle_{A P}=\left\langle f, e^{i \lambda t} A^{*} x\right\rangle_{A P}=\left(a(\lambda, f), A^{*} x\right)_{H}=\left\langle g, e^{i \lambda t} x\right\rangle_{A P}=(a(\lambda, g), x)_{H} .
$$

This implies that $A a(\lambda, f)=a(\lambda, g)$, so that $f \in D(\mathcal{A})$ and $\mathcal{A} f=g$. Finally, we show that $\sigma(\mathcal{A})=\sigma(A)$. If $\lambda \in \rho(A)$, then the operator $\mathcal{B}$ defined by $(\mathcal{B} f)(t)=$ $\sum_{\xi \in \sigma(f)}(\lambda-A)^{-1} a(\xi, f) e^{i \xi t}$ is easily seen to be the bounded inverse of $(\lambda-\mathcal{A})$, hence $\lambda \in \rho(\mathcal{A})$, or $\sigma(\mathcal{A}) \subset \sigma(A)$.

Conversely, if $\lambda \in \rho(\mathcal{A})$, then $\mathcal{A}-\lambda$ has a dense range and satisfies

$$
\|(\mathcal{A}-\lambda) f\|_{A P} \geq \delta\|f\|_{A P}
$$


for some $\delta>0$ and all $f \in D(\mathcal{A})$. This implies that $(A-\lambda)$ has a dense range and $\|(A-\lambda) x\|_{H} \geq \delta\|x\|_{H}$ for all $x \in D(A)$, so that $\lambda \in \rho(A)$.

Below we denote by $L=\mathcal{D}-\mathcal{A}$ the operator on $A P(\mathbf{R}, H)$ defined by $D(L)=$ $D(\mathcal{D}) \cap D(\mathcal{A})$ and $L f=\mathcal{D} f-\mathcal{A} f$ for all $f \in D(L)$.

Lemma 4. The operator $L=\mathcal{D}-\mathcal{A}$ is densely defined and closable.

Proof. Since $D(\mathcal{D})$ and $D(\mathcal{A})$ contain linear combinations of functions $e^{i \lambda t} x, \lambda \in$ $\mathbf{R}, x \in D(A)$, it follows that $L$ is densely defined. For $v(t)=\sum_{k=1}^{n} e^{\lambda_{k} t} x_{k}$ with $\lambda_{k} \in \mathbf{R}, x_{k} \in D\left(A^{*}\right)$, let $K v=\sum_{k=1}^{n}\left[\left(i \lambda_{k}\right)-A^{*}\right] x_{k} e^{i \lambda_{k} t}$. It is easily seen that

$$
\langle L f, v\rangle_{A P}=\langle f, K v\rangle_{A P}
$$

for each $f=\sum_{j=1}^{m} y_{j} e^{i \gamma_{j} t}, y_{j} \in D(A)$. Hence, $K v=L^{*} v$, so that $L^{*}$ is densely defined. This implies that $L$ is closable (and its closure is $L^{* *}$ ).

Below, we denote by $(\mathcal{D}-\mathcal{A})^{-}$the closure of $\mathcal{D}-\mathcal{A}$.

For every closed subset $\Lambda \subset \mathbf{R}$, we denote by $M(\Lambda)$ a subspace of $A P(\mathbf{R}, H)$ consisting of functions $g$ such that $\sigma(g) \subset \Lambda$.

Lemma 5. Let $\Lambda$ be a closed non-empty subset of $\mathbf{R}$. Then

(i) $M(\Lambda)$ is a closed invariant subspace with respect to $S(t), \mathcal{D}, \mathcal{A}$;

(ii) $\sigma(\mathcal{D} \mid M(\Lambda))=i \Lambda, \sigma(\mathcal{A} \mid M(\Lambda))=\sigma(A)$;

(iii) $\mathcal{D} \mid M(\Lambda)$ is bounded if (and only if) $\Lambda$ is compact.

Proof. (i) It is obvious that $M(\Lambda)$ is linear and invariant with respect to $S(t), \mathcal{D}$ and $\mathcal{A}$. Suppose $g_{n} \in M(\Lambda)$ and $\left\|g_{n}-g\right\|_{A P} \rightarrow 0$ as $n \rightarrow \infty$. This implies $\left\|a\left(\lambda, g_{n}\right)-a(\lambda, g)\right\|_{H} \rightarrow 0$. Since $\sigma\left(g_{n}\right) \subset \Lambda$, we have $a\left(\lambda, g_{n}\right)=0$ for all $\lambda \notin \Lambda$, which implies $a(\lambda, g)=0$ for all $\lambda \notin \Lambda$, or $\sigma(g) \subset \Lambda$. Hence $M(\Lambda)$ is closed.

(ii) If $\lambda \in \Lambda, x \in H$, then $h(t)=e^{i \lambda t} x \in D(\mathcal{D}) \cap M(\Lambda)$ and $\mathcal{D} h=i \lambda h$. Hence $i \lambda \in \sigma\left(\left.\mathcal{D}\right|_{M(\Lambda)}\right)$, which implies $i \Lambda \subset \sigma\left(\left.\mathcal{D}\right|_{M(\Lambda)}\right)$.

Suppose now that $\lambda_{0} \notin \Lambda$. Define, for $\lambda_{k} \in \Lambda, x_{k} \in H$,

$$
R\left(\sum_{k=1}^{n} e^{i \lambda_{k} t} x_{k}\right)=\sum_{k=1}^{n}\left(i \lambda_{k}-i \lambda_{0}\right)^{-1} e^{i \lambda_{k} t} x_{k} .
$$

It is clear that

$$
\begin{gathered}
\left\|R \sum_{k=1}^{n} e^{i \lambda_{k} t} x_{k}\right\|_{A P}^{2}=\left\|\sum_{k=1}^{n}\left(i \lambda_{k}-i \lambda_{0}\right)^{-1} e^{i \lambda_{k} t} x_{k}\right\|_{A P}^{2} \\
=\sum_{k=1}^{n}\left|\left(i \lambda_{k}-i \lambda_{0}\right)^{-1}\right|^{2}\left\|x_{k}\right\|^{2} \\
\left.\leq \sup _{\lambda \in \Lambda}\left|\lambda-\lambda_{0}\right|^{-1}\right)^{2}\left\|\sum_{k=1}^{n} e^{i \lambda_{k} t} x_{k}\right\|_{A P}^{2}
\end{gathered}
$$

hence $R$ can be extended to a bounded operator on $M(\Lambda)$. It is easily verified that $R$ is the inverse to $\left.\left(\mathcal{D}-i \lambda_{0}\right)\right|_{M(\Lambda)}$, hence $i \lambda_{0} \notin \sigma\left(\left.\mathcal{D}\right|_{M(\Lambda)}\right)$. The proof of $\sigma(\mathcal{A} \mid M(\Lambda))=\sigma(A)$ is analogous to that of $\sigma(\mathcal{A})=\sigma(A)$ in Lemma 3 .

(iii) The operator $\mathcal{D} \mid M(\Lambda)$, being skew self-adjoint, is bounded if and only if its spectrum, $i \Lambda$, is compact.

Assume that $f \in A P(\mathbf{R}, H)$. A function $u \in A P(\mathbf{R}, H)$ is called a mild solution of $(1)$ if $u \in D\left((\mathcal{D}-\mathcal{A})^{-}\right)$and $(\mathcal{D}-\mathcal{A})^{-} u=f$. The space $M(\Lambda)$ is called regularly 
admissible (w.r.t. (1)) if for every $f \in M(\Lambda)$, (1) has a unique mild solution $u$ in $M(\Lambda)$.

3.

Let $\Lambda$ be a closed subset of $\mathbf{R}$. If follows from Lemma $5(\mathrm{i})$ that $M(\Lambda)$ is invariant under $(\mathcal{D}-\mathcal{A})^{-}$, so that $(\mathcal{D}-\mathcal{A})^{-} \mid M(\Lambda)$ is defined. Assume that $M(\Lambda)$ is regularly admissible. Then we define a linear operator $K_{\Lambda}$ on $M(\Lambda)$, called the solution operator, by putting $K_{\Lambda} f=u$, where $u$ is the unique (mild) solution in $M(\Lambda)$ of (11). A standard argument, using the Closed Graph Theorem, shows that $K_{\Lambda}$ is a bounded operator on $M(\Lambda)$. Moreover, $(\mathcal{D}-\mathcal{A})^{-} u=(\mathcal{D}-\mathcal{A})^{-} K_{\Lambda} f=f$ (for all $f$ in $M(\Lambda)$ ). Therefore the operator $(\mathcal{D}-\mathcal{A})^{-} \mid M(\Lambda)$ is invertible (with the inverse equal to $K_{\Lambda}$ ). In particular, for every $\lambda_{0} \in \Lambda$ and $y \in H$, there exists a unique $x \in H$ such that $e^{i \lambda_{0} t} x$ is the unique mild solution in $M(\Lambda)$ of (1), with $f(t)=e^{i \lambda_{0} t} y$, which implies that for every $y \in H$ there exists a unique $x \in H$ such that $\left(i \lambda_{0}-A\right) x=y$, i.e. $\left(i \lambda_{0}-A\right)$ is invertible. Thus, $\sigma(A) \cap i \Lambda=\emptyset$. From this the following lemma, which is a version of [8, Theorem 3.1, follows.

Lemma 6 (cf. [8, Theorem 3.1). Let $\Lambda$ be a non-empty closed subset of $\mathbf{R}$. Then $M(\Lambda)$ is regularly admissible if and only if $(\mathcal{D}-\mathcal{A})^{-} \mid M(\Lambda)$ is invertible. If $M(\Lambda)$ is regularly admissible, then $i \Lambda \cap \sigma(A)=\emptyset$.

If $\Lambda$ is compact, then $\mathcal{D} \mid M(\Lambda)$ is bounded and, therefore, $(\mathcal{D}-\mathcal{A})^{-} \mid M(\Lambda)$ is invertible whenever $\sigma(\mathcal{D} \mid M(\Lambda)) \cap \sigma(\mathcal{A} \mid M(\Lambda))=\emptyset$, or $i \Lambda \cap \sigma(A)=\emptyset$ (see [1). Moreover, $K_{\Lambda}$ is given by the following analog of the Krein-Rosenblum integral formula (cf. [8, p. 397, 1]):

$$
K_{\Lambda}=\frac{1}{2 \pi i} \int_{\Gamma}\left(\lambda-\mathcal{A}_{M}\right)^{-1}\left(\lambda-\mathcal{D}_{M}\right)^{-1} d \lambda
$$

where $\Gamma$ is a Cauchy contour which surrounds $\sigma\left(\mathcal{D}_{M}\right)(=i \Lambda)$ and is separated from $\sigma\left(A_{M}\right)(=\sigma(A))$, and $\mathcal{A}_{M}$ and $\mathcal{D}_{M}$ are restrictions of $\mathcal{A}$ and $\mathcal{D}$, respectively, to $M(\Lambda)$. Thus, the following analog of ([8, Theorem 3.3-i) holds.

Lemma 7 ([8], Theorem 3.3-i). If $\Lambda$ is compact and $i \Lambda \cap \sigma(A)=\emptyset$, then $M(\Lambda)$ is regularly admissible and the solution operator $K_{\Lambda}$ is given by (4).

Assume that $i \Lambda \cap \sigma(A)=\emptyset$. In general, this condition does not imply that $M(\Lambda)$ is regularly admissible. Let $\Lambda_{\alpha}, \alpha \in \Omega$, be a family of compact subsets of $\Lambda$ such that $\operatorname{span}\left\{M\left(\Lambda_{\alpha}\right): \alpha \in \Omega\right\}$ is dense in $M(\Lambda)$. Then from $\Lambda \cap \sigma(A)=\emptyset$ it follows that $\Lambda_{\alpha} \cap \sigma(A)=\emptyset$ for all $\alpha$. According to Lemma 7, each subspace $M\left(\Lambda_{\alpha}\right)$ is regularly admissible. Let $K_{\Lambda_{\alpha}}$ be the solution operator on $M\left(\Lambda_{\alpha}\right)$. From the uniqueness of $K_{\Lambda_{\alpha}}$ it follows that if $\Lambda_{\alpha} \subset \Lambda_{\beta}$, then $K_{\Lambda_{\beta}} \mid M\left(\Lambda_{\alpha}\right)=K_{\Lambda_{\alpha}}$. Therefore, one can correctly define an operator $K_{0}$ with dense domain $D\left(K_{0}\right)=\operatorname{span}\left\{M\left(\Lambda_{\alpha}\right): \alpha \in \Omega\right\}$, by putting $K_{0} \mid M\left(\Lambda_{\alpha}\right)=K_{\Lambda_{\alpha}}$. If $M(\Lambda)$ is regularly admissible and $K_{\Lambda}$ is the corresponding solution operator, then $K_{\Lambda} \mid M\left(\Lambda_{\alpha}\right)=K_{\Lambda_{\alpha}}$, hence $\sup _{\alpha \in \Omega}\left\|K_{\Lambda_{\alpha}}\right\|=$ $\left\|K_{\Lambda}\right\|<\infty$. Conversely, if $\sup _{\alpha}\left\|K_{\Lambda_{\alpha}}\right\|=L<\infty$, then $\left\|K_{0}\right\| \leq L$ so that $K_{0}$ can be extended by continuity to a bounded operator $K_{\Lambda}$ on $M(\Lambda)$, which is the inverse of $(\mathcal{D}-\mathcal{A})^{-} \mid M(\Lambda)$. Thus, the following statement, which is a version of $[8$, Theorem 2.2 and Theorem 3.4, holds.

Lemma 8. Assume that $i \Lambda \cap \sigma(A)=\emptyset$. Let $\Lambda_{\alpha}, \alpha \in \Omega$, be a family of compact subsets such that $\operatorname{span}\left\{M\left(\Lambda_{\alpha}\right): \alpha \in \Omega\right\}$ is dense in $M(\Lambda)$. Then $M(\Lambda)$ is regularly admissible if and only if $\sup _{\alpha \in \Omega}\left\|K_{\Lambda_{\alpha}}\right\|<\infty$. 
4.

Proof of Theorem 2. In light of Lemma 6, we can assume that $\sigma(A) \cap i \Lambda=\emptyset$. Let $\lambda_{k} \in \Lambda$ and $\Lambda_{\alpha}=\left\{\lambda_{1}, \lambda_{2}, \ldots, \lambda_{n}\right\}$. By Lemma [7, $M\left(\Lambda_{\alpha}\right)$ is regularly admissible. Let $K_{\Lambda_{\alpha}}: M\left(\Lambda_{\alpha}\right) \rightarrow M\left(\Lambda_{\alpha}\right)$ be the corresponding solution operator. Consider the function $g(t)=\sum_{k=1}^{n} e^{i \lambda_{k} t} x_{k}$, where $x_{k}, k=1,2, \ldots, n$, are arbitrary vectors in $H$. It is directly verified that $u(t):=\sum_{k=1}^{n} e^{i \lambda_{k} t}\left(i \lambda_{k}-A\right)^{-1} x_{k}$ is a (classical) solution in $M\left(\Lambda_{\alpha}\right)$ of equation $u^{\prime}(t)=A u(t)+g(t)$, hence $K_{\Lambda_{\alpha}} g=\sum_{k=1}^{n} e^{i \lambda_{k} t}\left(i \lambda_{k}-A\right)^{-1} x_{k}$. According to Lemma $8, M(\Lambda)$ is regularly admissible if and only if $\sup _{\alpha}\left\|K_{\Lambda_{\alpha}}\right\|<$ $\infty$, that is, if and only if there exists $L>0$ such that

$$
\left\|\sum_{k=1}^{n} e^{i \lambda_{k} t}\left(\lambda_{k}-A\right)^{-1} x_{k}\right\|_{A P} \leq L\left\|\sum_{j=1}^{n} e^{i \lambda_{j} t} x_{j}\right\|_{A P}
$$

for every $x_{j} \in H, \lambda_{j} \in \Lambda, 1 \leq j \leq n$. By Parseval's equality

$$
\begin{aligned}
\left\|\sum_{k=1}^{n} e^{i \lambda_{k} t}\left(i \lambda_{k}-A\right)^{-1} x_{k}\right\|_{A P}^{2} & =\sum_{k=1}^{n}\left\|\left(i \lambda_{k}-A\right)^{-1} x_{k}\right\|_{H}^{2}, \\
\left\|\sum_{j=1}^{n} e^{i \lambda_{j} t} x_{j}\right\|_{A P}^{2} & =\sum_{j=1}^{n}\left\|x_{j}\right\|_{H}^{2} .
\end{aligned}
$$

Hence, (5) is equivalent to

$$
\sum_{k=1}^{n}\left\|\left(i \lambda_{k}-A\right)^{-1} x_{k}\right\|_{H}^{2} \leq L \sum_{j=1}^{n}\left\|x_{j}\right\|_{H}^{2},
$$

for all $x_{1}, x_{2}, \ldots, x_{n}$ in $H$ and $\lambda_{1}, \ldots, \lambda_{n}$ in $\Lambda$.

Therefore, it remains to show that (2) and (6) are equivalent, which is obvious.

5.

Let $\Lambda_{1}=\{2 \pi k: k \in \mathbf{Z}\}$. Then $M\left(\Lambda_{1}\right)$ can be naturally identified with the space $L^{2}([0,1], H)$.

Corollary 9. The following are equivalent:

(i) For every function $f \in L^{2}([0,1], H)$, there exists a unique mild solution $u \in$ $L^{2}([0,1], H)$ of $(1)$;

(ii) $i 2 \pi k \in \rho(A)$ for all $k \in \mathbf{Z}$ and $\sup _{k \in \mathbf{Z}}\left\|(i 2 \pi k-A)^{-1}\right\|<\infty$.

Now let $A$ be the generator of a $C_{0}$-semigroup $T(t)$ on $H$. Assuming that $M\left(\Lambda_{1}\right)$ is regularly admissible, we show that mild solutions in our definition are mild solutions in the standard sense of the theory of $C_{0}$-semigroups (see e.g. [6]), i.e. the following equation holds:

$$
u(t)=T(t-s) u(s)+\int_{s}^{t} T(t-\tau) f(\tau) d \tau(t \geq s) .
$$

Proposition 10. Under the conditions of Corollary 9 , for every $f \in L^{2}([0,1], H)$ the unique mild solution $u$ in $L^{2}([0,1], H)$ is a continuous 1-periodic function and satisfies (71). 
Proof. Let $f_{n}=\sum_{k=-n}^{n} e^{i 2 \pi k t} \hat{f}(k)$ and $u_{n}=\sum_{k=-n}^{n} e^{i 2 \pi k t}(i 2 \pi-A)^{-1} \hat{f}(k)$, where $\hat{f}(k)$ and $\hat{u}(k)$ are Fourier coefficients of $f$ and $u$, respectively. Using the well-known property

$$
A \int_{0}^{t} T(s) x d s=T(t) x-x
$$

(which is valid for arbitrary semigroups $T(t)$, with generator $A$, and arbitrary $x \in$ $H)$, we obtain

$$
(A-i 2 \pi k) \int_{0}^{t} T(s) e^{-i 2 \pi k s} \hat{f}(k) d s=e^{-i 2 \pi k t} T(t) \hat{f}(k)-\hat{f}(k),
$$

which implies

$$
\begin{gathered}
e^{i 2 \pi k t}(i 2 \pi k-A)^{-1} \hat{f}(k) \\
=T(t)\left[(i 2 \pi k-A)^{-1} \hat{f}(k)\right]+\int_{0}^{t} T(t-\tau) e^{i 2 \pi k \tau} \hat{f}(k) d \tau .
\end{gathered}
$$

From (8) it follows that

$$
u_{n}(t)=T(t) u_{n}(0)+\int_{0}^{t} T(t-\tau) f_{n}(\tau) d \tau
$$

i.e. $u_{n}$ is a mild solution of the equation $u^{\prime}(t)=A u(t)+f_{n}(t)$ in the traditional sense (of (7)). From the last identity it follows that

$$
v_{n}:=[I-T(1)] u_{n}(0)=\int_{0}^{1} T(1-s) f_{n}(s) d s,
$$

so that $\left\|v_{n}-v_{m}\right\| \leq \sup _{0 \leq t \leq 1}\|T(t)\|\left\|f_{n}-f_{m}\right\|_{L^{2}}$, i.e. $v_{n}$ converges to some $v \in H$. Furthermore

$$
\begin{gathered}
T(1) u_{n}(0)=\int_{0}^{1} T(1-t) T(t) u_{n}(0) \\
=\int_{0}^{1} T(1-t) u_{n}(t) d t-\int_{0}^{1} T(1-t) \int_{0}^{t} T(t-\tau) f_{n}(\tau) d \tau d t
\end{gathered}
$$

which also implies that $w_{n}:=T(1) u_{n}(0)$ is a convergent sequence. Therefore, $u_{n}(0)=v_{n}+w_{n}$ converges to some vector $u_{0} \in H$. From

$$
\begin{gathered}
\left\|u_{n}(t)-u_{m}(t)\right\| \\
\leq \sup _{0 \leq t \leq 1}\|T(t)\|\left[\left\|u_{n}(0)-u_{m}(0)\right\|+\left\|f_{n}-f_{m}\right\|_{L^{2}}\right], 0 \leq t \leq 1,
\end{gathered}
$$

it follows that $u_{n}$ converges to $u$ uniformly on $[0,1]$, so that $u$ is a continuous 1-periodic function. The equality (7) is now immediate.

In conclusion we remark that the presented approach is directly applicable to more general classes of differential, integro-differential and functional-differential equations in a Hilbert space. The details are to be given elsewhere.

\section{ACKNOWLEDGMENT}

The author would like to thank the anonymous referee for pointing out some inaccuracies in the original version of the paper. 


\section{REFERENCES}

[1] W. Arendt, F. RÄBiger And A. Sourour, Spectral properties of the operator equation AX+ $X B=Y$, Quart. J. Math, 45(1994), 133-149. MR1280689 (95g:47060)

[2] L. Gearhart, Spectral theory for contraction semigroups on Hilbert spaces, Trans. Amer. Math. Soc. 236(1978), 385-394. MR0461206 (57:1191)

[3] I.W. Herbst, The spectrum of Hilbert space semigroup, J. Operator Theory 10 (1983), 87-94. MR0715559(84m:47052)

[4] J.S. Howland, On a theorem of Gearhart, Integral Equations and Operator Theory 7 (1984), 138-142. MR0802373 (87b:47044)

[5] B.M. Levitan AND V.V. ZhiKOv, Almost Periodic Functions and Differential Equations, Cambridge Univ. Press, Cambridge, 1982. MR0690064 (84g:34004)

[6] R. Nagel (ED.), One-parameter Semigroups of Positive Operators, Lecture Notes in Math, Vol. 1184, Springer-Verlag, Berlin, 1984. MR0839450 (88i:47022)

[7] J. PRüss, On the spectrum of $C_{0}$-semigroups, Trans. Amer. Math. Soc. 284 (1984), 847-857. MRO0743749 (85f:47044)

[8] Vu Quoc Phong And E. Schüler, The operator equation $A X-X B=C$, admissibility, and asymptotic behavior of differential equations, J. Differential Equations, 145(1998), 394-419. MR.1621042 (99h:34081)

Department of Mathematics, Ohio University, Athens, Ohio 45701

E-mail address: quu@math.ohiou.edu 